\title{
ON A FORMULA CONCERNING STOCHASTIC DIFFERENTIALS
}

\section{KIYOSI ITÔ}

In his previous paper [1]') the author has stated a formula') concering stochastic differentials with the outline of the proof. The aim of this paper is to show this formula in details in a little more general form (Theorem 6).

1. Definitions. Throughout this paper we assume that all stochastic processes $^{3 !} \xi(t, \omega), \eta(t, \omega), a(t, \omega), b(t, \omega)$, etc. are measurable in variables $t$ and $\omega$. A system of $r$ one-dimensional Brownian motions independent of each other is called an $r$-dimensional Brownian motion.

Given two system of stochastic processes:

$$
\xi=\left\{\xi_{\lambda}(t, \omega), \lambda \in \Lambda\right\}, \quad \eta=\left\{\eta_{\mu}(t, \omega), \mu \in M\right\} .
$$

We say that $\xi$ has the property $\alpha$ with regard to $\eta$ in $u \leqq t \leqq v$, if, for any $t$, the following two systems of random variables are independent of one another:

$$
\left\{\begin{array}{l}
\varphi_{t}=\left\{\xi_{\lambda}(\tau, \omega), \lambda \in \Lambda, \eta_{u}(\tau, \omega), \mu \in M, u \leqq \tau \leqq t\right\} \\
\phi_{t}=\left\{\eta_{\mu}(\sigma, \omega)-\eta_{\mu}(t, \omega), \mu \in M, t \leqq \sigma \leqq v\right\} .
\end{array}\right.
$$

Now we shall state an outline ${ }^{4)}$ of a stochastic integral of the form:

$$
\int_{s}^{t} \xi(\tau, \omega) d \beta(\tau, \omega), \quad u \leqq s \leqq t \leqq v, \quad \omega \in \Omega_{1},
$$

where $\beta(t, \omega)$ is a one-dimensional Brownian motion and $\Omega_{1}$ is a measurable subset of $\Omega$. We shall set the two conditions on $\xi$;

$$
\xi(t, \omega) \text { has the property } \alpha \text { concerning } \beta(t, \omega) \text { in } u \leqq t \leqq v \text {, }
$$

$$
\int_{u}^{v} \xi(\tau, \omega)^{2} d \tau \text { for almost all } \omega \in \Omega_{1} \text {. }
$$

Received April 16, 1951.

1) The number in [ ] refers to the Reference at the end of this paper.

2) Theorem 1.1 in [1].

3) In the analytical theory of probability any stochastic process is expressed as a function of the time parameter $t$ and the probability parameter $\omega$ which runs over a probability space $\Omega(P), P$ being the probability distribution.

4. Cf. [2] concerning the details. 
Case 1. When $\xi$ is uniformly stepwise, that is when there exists a system of time-points :

$$
u=s_{0}<s_{1}<\ldots<s_{n}=v
$$

such that

$$
\xi(\tau, \omega)=\xi\left(s_{i-1}, \omega\right), s_{i-1} \leqq \tau<s_{i}, \quad i=1,2, \ldots, n,
$$

we define

$$
\begin{aligned}
& \int_{s}^{t} \xi(\tau, \omega) d \beta(\tau, \omega)=\sum_{i=k+1}^{l-1} \xi\left(s_{i-1}, \omega\right)\left(\beta\left(s_{i}, \omega\right)-\beta\left(s_{i-1}, \omega\right)\right) \\
& +\xi\left(s_{k-1}, \omega\right)\left(\beta\left(s_{k}, \omega\right)-\beta(s, \omega)\right)+\xi\left(s_{l-1}, \omega\right)\left(\beta(t, \omega)-\beta\left(s_{l-1}, \omega\right)\right) \\
& \quad\left(s_{k-1} \leqq s<s_{k}, s_{l-1} \leqq t<s_{l}\right) .
\end{aligned}
$$

Case 2. When

$$
\int_{\Omega} \int_{u}^{v} \xi(t, \omega)^{2} d t P(d \omega)<\infty,
$$

there exists a sequence of uniformly stepwise processes $\xi_{n}(t, \omega), n=1,2, \ldots$ whose value at any time-point $t$ is a $B$-measurable function of $\xi(\tau, \omega), u \leqq \tau \leqq t$, such that

$$
\int_{\Omega} \int_{u}^{v}\left(\xi_{n}(t, \omega)-\xi(t, \omega)\right)^{2} d t P(d w)<8^{-n}
$$

We define

$$
\int_{s}^{t} \hat{\xi}(\tau, \omega) d \beta(\tau, \omega)=\lim _{n} \int_{s}^{t} \xi_{n}(\tau, \omega) d \beta(\tau, \omega) .
$$

As was proved in our previous paper [2], the sequence:

$$
\int_{s}^{t} \xi_{n}(\tau, \omega) d \beta(\tau, \omega), n=1,2, \ldots,
$$

is uniformly convergent in $u \leqq s \leqq t \leqq v$ for almost all $\omega$, and the definition is independent of the special choice of the sequence $\left\{\xi_{n}(t, \omega)\right\}$.

Case 3. Now we shall consider the general case. Put

$$
\phi_{n}(\lambda)= \begin{cases}1 & (|\lambda| \leqq n) \\ 0 & (|\lambda|>n)\end{cases}
$$

and

$$
\xi_{n}(t, \omega)=\phi_{n}\left(\int_{u}^{t} \xi(\tau, \omega)^{2} d t\right) \xi(t, \omega), \quad n=1,2, \ldots
$$

Then $\xi_{n}(t, \omega)$ satisfies the conditions (1.6) and (C.1), and so their stochastic integrals are well defined. Since $\xi n(t, \omega)=\xi(t, \omega), u \leqq t \leqq v$, for a sufficiently large $n$ for almost all $\omega \in \Omega_{1}$ by (C.2), we define naturally 


$$
\int_{s}^{t} \xi(\tau, \omega) d \beta(\tau, \omega)=\lim _{n} \int_{s}^{t} \xi_{n}(\tau, \omega) d \beta(\tau, \omega)
$$

2. Preliminary properties of stochastic integrals. In the following Theorems 1,3 , and 4 we shall assume that $\xi(t, \omega)$ satisfies the conditions (C. 1 ) and (C.2).

THEOREM 1. The stochastic integral (1.3) is continuous in $s$, $t$ for almost all $\omega \in \Omega_{\text {. }}$.

THEOREM 2. If each of $\xi(t, \omega)$ and $\eta(t, \omega)$ satisfies (C. 1) and (C. 2), and if the system $\{\xi(t, \omega), \eta(t, \omega)\}$ has the property a with regard to the Brownian motion $\beta$, then $\zeta(t, \omega) \equiv a \xi(t, \omega)+b \eta(t, \omega)$ ( $a, b$ being constants) satisfies (C. 1$)$ and (C.2) and we obtain

$$
\int_{s}^{t}\left(a \xi(\tau, \omega)+b r_{i}(\tau, \omega)\right) d \beta(\tau, \omega)=a \int_{0}^{t} \xi(\tau, \omega) d \beta(\tau, \omega)+b \int_{s}^{t} \eta(\tau, \omega) d \beta(\tau, \omega)
$$

for $u \leqq t \leqq s \leqq v$ for almost all $\omega \in \Omega_{1}$.

THEOREM 3. We have

$$
\int_{s_{1}}^{s_{2}} \xi(\tau, \omega) d \beta(\tau, \omega)+\int_{s_{2}}^{s_{3}} \xi(\tau, \omega) d \beta(\tau, \omega)=\int_{s_{1}}^{s_{3}} \xi(\tau, \omega) d \beta(\tau, \omega)
$$

for $u \leqq s_{1} \leqq s_{2} \leqq s_{3} \leqq v$ for almost all $\omega \in \Omega_{1}$.

THEOREM 4. If (1.6) is satisfied, then we have

$$
\begin{aligned}
& \frac{c^{2}}{2} \operatorname{Pr}\left\{\sup _{u \leqq s \geqq t \leqq v} \mid \int_{s}^{t} \xi(\tau, \omega) d \beta(\tau, \omega) \geqq 2 c\right\} \\
& \quad \leqq c^{2} \operatorname{Pr}\left\{\sup _{u \leqq t \leqq v}\left|\int_{u}^{t} \xi(\tau, \omega) d \beta(\tau, \omega)\right| \geqq c\right\} \\
& \quad \leqq \int_{\Omega}\left(\int_{u}^{v} \xi(t, \omega) d \beta(t, w)\right)^{2} P(d \omega)=\int_{\Omega} \int_{u}^{v} \xi(t, \omega)^{2} d t P(d \omega) .
\end{aligned}
$$

THEOREM 5. If each of $\xi_{n}(t, \omega), n=1,2, \ldots$, satisfies (C. 1) and (C.2) and if the system $\left\{\xi_{n}(t, \omega), n=1,2, \ldots, \infty\right\}$ has the property $\alpha$ with regard to $\beta$ in $u \leqq t \leqq v$, and further if

$$
\int_{u}^{v}\left(\xi_{n}(t, \omega)-\xi_{\infty}(t, \omega)\right)^{2} d t \rightarrow 0
$$

for almost all $\omega \in \Omega_{1}$, then

$$
\sup _{u \cong s<t \leqq v}\left|\int_{s}^{t} \xi_{n}(\tau, \omega) d \beta(\tau, \omega)-\int_{s}^{t} \xi_{\infty}(\tau, \omega) d \beta(\tau, \omega)\right|
$$

tends to 0 in probability over $\Omega_{1}$.

Since Theorems 1,2, 3 and 4 follow at once from the properties of the stochastic integral established in [2], we shall here prove Theorem 5 only. 
Since we have

$$
\int_{u}^{v} \xi_{n}(t, \omega)^{2} d t \rightarrow \int_{u}^{v} \xi_{\infty}(t, \omega)^{2} d t
$$

for almost all $\omega \in \Omega_{1}$ by the assumption (2.5) and since there exists $M=M(\varepsilon)$ for any $\varepsilon>0$ such that

$$
\operatorname{Pr}\left\{\omega \in \Omega_{1}, \int_{u}^{v} \xi_{\infty}(t, \omega)^{2} d t<M\right\}>P\left(\Omega_{1}\right)-\varepsilon
$$

by the assumption that $\xi_{\infty}(t, \omega)$ satisfies (C. 2$)$, there exists $N_{1}=N_{1}(\varepsilon)$ for any $\varepsilon>0$

$$
\operatorname{Pr}\left\{\omega \in \Omega_{1}, \int_{u}^{v} \xi_{n}(t, \omega)^{2} d t<M, N_{1}<n \leqq \infty\right\}>P\left(\Omega_{1}\right)-2 \varepsilon .
$$

Put

$$
\xi_{n}^{M}(t, \omega)=\phi_{M}\left(\sup _{n \triangleq k \equiv \infty} \int_{u}^{t} \xi_{k}(\tau, \omega)^{2} d t\right) \xi_{n}(t, \omega), n=1,2, \ldots, \infty,
$$

where $\phi_{M}$ is defined by (1.9). Then it follows from (2.7) that

$$
\operatorname{Pr}\left\{\omega \in \Omega_{1}, \xi_{n}^{M}(t, \omega)=\xi_{n}(t, \omega), u \leqq t \leqq v, N_{1}<n \leqq \infty\right\}>P\left(\Omega_{1}\right)-2 \varepsilon .
$$

Since we have

$$
\begin{gathered}
\left.\int_{u}^{v}\left(\xi_{n}^{M}(t, \omega)-\xi_{\infty}^{M}(t, \omega)\right)^{2} d t \leqq \int_{u}^{v} \xi_{n}(t, \omega)-\xi_{\infty}(t, \omega)\right)^{2} d t \rightarrow 0, \\
\int_{u}^{v}\left(\xi_{n}^{M}(t, \omega)-\xi_{\infty}^{M}(t, \omega)\right)^{2} d t \leqq 2 \int_{u}^{v} \xi_{n}^{M}(t, \omega)^{2} d t+2 \int_{u}^{v} \xi_{\infty}^{M}(t, \omega)^{2} d t<4 M,
\end{gathered}
$$

we obtain

$$
\int_{\Omega} \int_{u}^{v}\left(\xi_{n}^{M}(t, \omega)-\xi_{\infty}^{M}(t, \omega)\right)^{2} d t P(d \omega) \rightarrow 0 .
$$

By Theorem 4 there exists $N_{2}=N_{2}(\varepsilon)$ for any $\varepsilon>0$ such that

$$
\operatorname{Pr}\left\{\sup _{u \leqq s \leqq t \leqq v}\left|\int_{s}^{t} \xi_{n}^{M I}(\tau, \omega) d \beta(\tau, \omega)-\int_{s}^{t} \xi_{\infty}^{M}(\tau, \omega) d \beta(\tau, \omega)\right|<\varepsilon\right\}>P\left(\Omega_{1}\right)-3 \varepsilon,
$$

which, combined with (2.8), proves our theorem.

3. A formula concerning stochastic differentials. Let $\beta \equiv\left(\beta^{i}(t, \omega), i=1\right.$, $2, \ldots, r)$ be an $r$-dimensional Brownian motion, and let the system:

$$
\left\{\xi^{i}(t, \omega), a^{i}(t, \omega), b_{j}^{i}(t, \omega), i=1,2, \ldots, n, j=1,2, \ldots, r\right\}
$$

have the property $\alpha$ with regard to $\beta$ in $u \leqq t \leqq v$. When we have

$$
\xi^{i}(s, \omega)-\xi^{i}(t, \omega)=\int_{t}^{s} a^{i}(\tau, \omega) d \tau+\int_{t}^{s} b_{j}{ }^{i}(\tau, \omega) d \beta^{j}(\tau, \omega),{ }^{5)} u \leqq t \leqq s \leqq v, 1 \leqq i \leqq n,
$$

5) We omit the summation sign $\sum_{j=1}^{r}$ according to the usual rule of tensor calculus. 
for almost all $\omega \in \Omega_{1}$, we write this relation in the differential form as follows:

$$
d \xi^{i}(t, \omega)=a^{i}(t, \omega) d t+b_{j}^{i}(t, \omega) d \beta^{j}(t, \omega), \quad u \leqq t \leqq v, \quad \omega \in \Omega_{1}, \quad 1 \leqq i \leqq n .
$$

THEOREM 6. Let $\xi^{i}(t, \omega), i=1,2, \ldots, n$, satisfy

$$
d \xi^{i}(t, \omega)=a^{i}(t, \omega) d t+b_{j}{ }^{i}(t, \omega) d \beta^{j}(t, \omega), \quad i=1,2, \ldots, n,
$$

and $G$ be an open subset of the $n$-space $R^{n}$ which contains all the points $\left(\xi^{i}(t, \omega)\right.$. $i=1,2, \ldots, n)$ for $u \leqq t \leqq v, \omega \in \Omega_{1}$.

Let $f\left(t, x^{1}, x^{2}, \ldots, x^{n}\right)$ be a continuous function defined in $u \leqq t \leqq v,\left(x^{1}\right.$. $\left.x^{2}, \ldots, x^{n}\right) \in G$, such that

$$
\left\{\begin{array}{l}
f_{0}\left(t, x^{1}, \ldots, x^{n}\right)=\frac{\partial f}{\partial t}\left(t, x^{1}, \ldots, x^{n}\right) \\
f_{i}\left(t, x^{1}, \ldots, x^{n}\right)=\frac{\partial f}{\partial x_{i}}\left(t, x^{1}, \ldots, x^{n}\right), i=1,2, \ldots, n, \\
f_{i j}\left(t, x^{1}, \ldots, x^{n}\right)=\frac{\partial^{2} f}{\partial x^{i} \partial x^{j}}\left(t, x^{1}, \ldots, x^{n}\right), i, j=1,2, \ldots, n .
\end{array}\right.
$$

are all continous.

Then $\eta(t, \omega) \equiv f\left(t, \xi^{1}(t, \omega), \ldots, \xi^{n}(t, \omega)\right)$ satisfies

$$
\begin{aligned}
d \eta(t, \omega)=\left(f_{0}(t, \xi)\right. & \left.+f_{i}(t, \xi) a^{i}(t, \omega)+\frac{1}{2} f_{i j}(t, \xi) b_{k}^{i}(t, \omega) b_{k}^{j}(t, \omega)\right) d t \\
& +f_{i}(t, \xi) b_{j}^{i}(t, \omega) d \beta^{j}(t, \omega),
\end{aligned}
$$

where $\xi \equiv\left(\xi^{1}(t, \omega), \xi^{2}(t, \omega), \ldots, \xi^{n}(t, \omega)\right)$.

LEMMA 1. For any stochastic process $\xi(t, \omega)$ satisfying

$$
\int_{u}^{v} \xi(t, \omega)^{2} d t<\infty, \quad \omega \in \Omega_{1},
$$

there exists a sequence of uniformly stepwise stochastic processes $\xi_{n}(t, \omega), n=1$. $2, \ldots$ whose value at any time-point $t$ is a B-measurable function of $\xi(\tau, \omega)$. $-\leqq t$, and such that

$$
\int_{u}^{v}|\xi n(t, \omega)-\xi(t, \omega)|^{2} d t \rightarrow 0
$$

for almost all $\omega \in \Omega_{1}$.

This Lemma follows immediately from Lemma 7.1 in [2].

Lemma 2. Let $\xi(t, \omega), \eta(t, \omega)$ be stochastic processes such that the system $\{\xi(t, \omega), \eta(t, \omega)\}$ has the property $\alpha$ with regard to a one-dimensional Brownian motion $\beta(t, \omega)$ in $u \leqq t \leqq v$ and that

$$
\int_{u}^{v} \hat{\xi}(t, \omega)^{2} d t<\infty, \quad \int_{u}^{v} \eta(t, \omega)^{2} d t<\infty
$$

for almost all $\omega \in \Omega_{1}$. Then we have 


$$
\begin{aligned}
\int_{u}^{v} \xi(t, \omega) d \beta(t, \omega) \int_{u}^{v} \eta(s, \omega) d \beta(s, \omega) \\
\quad=\int_{u}^{v} \xi(t, \omega) \int_{u}^{t} \eta(s, \omega) d \beta(s, \omega) d \beta(t, \omega) \\
\quad+\int_{u}^{v} \eta(s, \omega) \int_{u}^{s} \xi(t, \omega) d \beta(t, \omega) d \beta(s, \omega)+\int_{u}^{v} \xi(t, \omega) \eta(t, \omega) d t
\end{aligned}
$$

for almost all.

Proof. Firstly we shall prove (3.8) in the case that both $\xi(t, \omega)$ and $\eta(t, \omega)$ are uniformly stepwise. Then we may assume that

$$
\xi(t, \omega)=\xi\left(u_{i-1}, \omega\right), \quad \eta(t, \omega)=\eta\left(u_{i-1}, \omega\right), \quad u_{i-1} \leqq t \leqq u_{i}, i=1,2, \ldots, n,
$$
where

$$
u=u_{0}<u_{1}<\ldots<u_{n}=v .
$$

The left side of (3.8) equals the following: $\left(u_{i j}=u_{i}+\frac{j}{N}\left(u_{i+1}-u_{i}\right)\right)$

$$
\begin{aligned}
& \sum_{i j p q} \int_{u_{i, j-1}}^{u_{i j}} \xi(t, \omega) d \beta(t, \omega) \int_{u_{p, q-1}}^{u_{p q}} \eta(s, \omega) d \beta(s, \omega) \\
& \quad=\sum_{u_{i j}>u_{p q}}+\sum_{u_{p q}>u_{i j}}+\sum_{p=i, q=j}=\int_{u}^{v} \xi(t, \omega) \int_{u}^{\lambda_{\eta} ! t} \eta(s, \omega) d \beta(s, \omega) d \beta(t, \omega) \\
& \quad+\int_{u}^{v} \eta(s, \omega) \int_{u}^{\lambda_{n}(s)} \xi(t, \omega) d \beta(t, \omega) d \beta(s, \omega) \\
& \quad+\sum_{i} \xi\left(u_{i-1}, \omega\right) \eta\left(u_{i-1}, \omega\right) \sum_{j=1}^{N}\left(\beta\left(u_{i j}, \omega\right)-\beta\left(u_{i, j-1}, \omega\right)\right)^{2}=I_{1}+I_{2}+I_{3},
\end{aligned}
$$

where $\lambda_{n}(t)$ is the maximum $u_{i j}$ which does not exceed $t$.

As $N \rightarrow \infty, I_{1}$ tends to the first term of the right side of (3.8) in probability by virtue of Theorem 5 , since we have

$$
\begin{aligned}
\int_{u}^{v}\left|\xi(t, \omega) \int_{u}^{\lambda_{n}(t)} \eta(s, \omega) d \beta(s, \omega)-\xi(t, \omega) \int_{u}^{t} \eta(s, \omega) d \beta(s, \omega)\right|^{2} d t \\
\quad=\int_{u}^{v}\left|\xi(t, \omega) \int_{\lambda_{n}(t)}^{t} \eta(s, \omega) d \beta(s, \omega)\right|^{2} d t \\
=\int_{u}^{v}\left|\xi(t, \omega) \eta\left(\lambda_{n}(t), \omega\right)\left(\beta(t, \omega)-\beta\left(\lambda_{n}(t), \omega\right)\right)\right|^{2} d t \rightarrow 0
\end{aligned}
$$

for almost all $\omega$. By the same reason we see that $I_{0}$ tends to the second term of the right side of $(3.8)$ in probability.

Since $\sum_{j=1}^{N}\left(\beta\left(u_{i j}, \omega\right)-\beta\left(u_{i, j-1}, \omega\right)\right)^{2} \rightarrow u_{i}-u_{i-1}$ (in probability), $I_{3}$ tends to the third term of the right side of (3.8).

Next we shall consider the general case. By Lemma 1 we shall construct $\xi_{n}(t, \omega), n=1,2, \ldots$, and $\eta_{n}(t, \omega), n=1,2, \ldots$ for $\xi(t, \omega)$ and $\eta(t, \omega)$ respec- 
tively. Since our Lemma 2 holds for uniformly stepwise processes as is proved above, we have

$$
\begin{aligned}
& \int_{u}^{v} \xi_{n}(t, \omega) d \beta(t, \omega) \int_{u}^{v} \eta_{n}(t, \omega) d \beta(s, \omega) \\
& \quad=\int_{u}^{v} \xi_{n}(t, \omega) \int_{u}^{t} \eta_{n}(s, \omega) d \beta(s, \omega) d \beta(t, \omega) \\
& \quad+\int_{u}^{v} \eta_{n}(s, \omega) \int_{u}^{s} \xi_{n}(t, \omega) d \beta(t, \omega) d \beta(s, \omega)+\int_{u}^{v} \xi_{n}(t, \omega) \eta_{n}(t, \omega) d t .
\end{aligned}
$$

Put

$$
\begin{array}{ll}
\zeta_{n}(t, \omega)=\int_{u}^{t} \eta_{n}(s, \omega) d \beta(s, \omega), & \zeta(t, w)=\int_{u}^{t} \eta(s, \omega) d \beta(s, \omega), \\
\rho_{n}(t, \omega)=\int_{u}^{t} \xi_{n}(s, \omega) d \beta(s, \omega), & \rho(t, \omega)=\int_{u}^{t} \eta(s, \omega) d \beta(s, \omega) .
\end{array}
$$

By taking adequate subsequences we see, by Theorem 5 , that $\zeta_{n}(t, \omega)$ and $\rho_{n}(t, \omega)$ converge uniformly in $t$ to $\zeta(t, \omega)$ and $\rho(t, \omega)$ respectively for almost all $\omega \in \Omega_{1}$. Therefore we have

$$
\begin{aligned}
\int_{u}^{v}\left|\xi_{n}(t, \omega) \zeta_{n}(t, \omega)-\xi(t, \omega) \zeta(t, \omega)\right|^{2} d t \\
\quad \leqq 2 \int_{u_{i}}^{v}\left|\xi_{n}(t, \omega)-\xi(t, \omega)\right|^{2}\left|\zeta_{n}(t, \omega)\right|^{2} d t \\
+2 \int_{u}^{v}|\xi(t, \omega)|^{2}\left|\zeta_{n}(t, \omega)-\zeta(t, \omega)\right|^{2} d t \rightarrow 0
\end{aligned}
$$

for almost all $\omega \in \Omega_{1}$, from which follows by Theorem 5

$$
\int_{u}^{v} \xi_{n}(t, \omega) \zeta_{n}(t, \omega) d \beta(t, \omega) \rightarrow \int_{u}^{v} \xi(t, \omega) \zeta(t, \omega) d \beta(t, \omega) \quad \text { (in probability). }
$$

Similaly we have

$$
\int_{u}^{v} \eta_{n}(s, \omega) \rho_{n}(s, \omega) d \beta(s, \omega) \rightarrow \int_{u}^{v} \eta(s, \omega) \rho(s, \omega) d \beta(s, \omega) \quad \text { (in probability). }
$$

Further we have

$$
\begin{aligned}
& \left|\int_{u}^{v} \xi_{n}(t, \omega) \eta_{n}(t, \omega) d t-\int_{u}^{v} \xi(t, \omega) \eta(t, \omega) d t\right| \\
& \quad \leqq \sqrt{\int_{u}^{v} \eta_{n}(t, \omega)^{2} d t \int_{u}^{v}(\xi n(t, \omega)-\xi(t, \omega))^{2} d t} \\
& +\sqrt{\int_{u}^{v} \xi(t, \omega) d t \int_{u}^{v}\left(\eta_{n}(t, \omega)-\eta(t, \omega)\right)^{2} d t} \rightarrow 0 .
\end{aligned}
$$

Thus our Lemma 2 is completely proved. 
By the same way as above, we obtain the following Lemmas 3 and 4 .

Lemma 3. Let $\xi(t, \omega)$ and $\eta(t, \omega)$ be stochastic processes such that the system $\{\xi(t, \omega), \eta(t, \omega)\}$ has the property $\alpha$ with regard to the two-dimensional Brownian motion $(\beta(t, \omega), \gamma(t, \omega))$ and that

$$
\int_{u}^{v} \xi(t, \omega)^{2} d t<\infty, \quad \int_{u}^{v} \eta(t, \omega)^{2} d t<\infty
$$

for almost all $\omega \in \Omega_{1}$.

Then we have

$$
\begin{aligned}
\int_{u}^{v} \xi(t, \omega) d \beta( & t, \omega) \int_{u}^{t} \eta(s, \omega) d \gamma(s, \omega) \\
& =\int_{u}^{v} \xi(t, \omega) \int_{u}^{t} \eta(s, \omega) d \gamma(s, \omega) d \beta(t, \omega) \\
& +\int_{u}^{v} \eta(s, \omega) \int_{u}^{s} \xi(t, \omega) d \beta(t, \omega) d \gamma(s, \omega)
\end{aligned}
$$

for almost all $\omega \in \Omega_{1}$.

Lemma 4. Let $a(t, \omega)$ and $b(t, \omega)$ be stochastic processes such that the system $a(t, \omega), b(t, \omega)$ has the property $\alpha$ with regard to a one-dimensional Brownian motion $\beta(t, \omega)$ and that

$$
\int_{u}^{v}|a(t, \omega)| d t<\infty, \quad \int_{u}^{v}|b(t, \omega)|^{2} d t<\infty
$$

for almost all $\omega \in \Omega_{1}$. Then we have

$$
\begin{aligned}
& \int_{u}^{v} a(t, \omega) d t \int_{u}^{v} b(s, \omega) d \beta(s, \omega) \\
& \quad=\int_{u}^{v} a(t, \omega) \int_{u}^{t} b(s, \omega) d \beta(s, \omega) d t+\int_{u}^{v} b(s, \omega) \int_{u}^{s} a(t, \omega) d t d \beta(s, \omega) .
\end{aligned}
$$

Lemia 5. Let $\xi^{i}(t,(0), i=1,2, \ldots, n$, be determined as in Theorem 6 . Then we have

$$
\left(\xi^{i}(s, \omega)-\xi^{i}(t, \omega)\right)\left(\xi^{j}(s, \omega)-\xi^{j}(t, \omega)\right)
$$

$$
\begin{aligned}
& =\int_{t}^{s}\left\{\left(\xi^{i}(\tau, \omega)-\xi^{i}(t, \omega)\right) a^{j}(\tau, \omega)+\left(\xi^{j}(\tau, \omega)-\xi^{j}(t, \omega)\right) a^{i}(\tau, \omega)+b_{k}^{i}(\tau, \omega) b_{k}^{j}(\tau, \omega)\right\} d \tau \\
& +\int_{t}^{j}\left\{\left(\xi^{i}(\tau, \omega)-\xi^{i}(t, \omega)\right) b_{k}^{j}(\tau, \omega)+\left(\xi^{j}(\tau, \omega)-\xi^{j}(t, \omega)\right) b_{k}^{i}(\tau, \omega)\right\} d \beta^{k}(\tau, \omega)
\end{aligned}
$$

for almost all $\omega \in \Omega_{1}$ and for $u \leqq t \leqq s \leqq v$. 
Proof. By the assumption we have

$$
\begin{aligned}
\left(\xi^{i}(s, \omega)-\xi^{i}(t, \omega)\right)\left(\xi^{i}(s, \omega)-\xi^{j}(t, \omega)\right) \\
\quad=\int_{t}^{s} a^{i}(\tau, \omega) d \tau \int_{t}^{s} a^{j}(\sigma, \omega) d \sigma+\int_{t}^{s} b_{k}^{i}(\tau, \omega) d \xi^{k}(\tau, \omega) \int_{t}^{s} a^{j}(\sigma, \omega) d \sigma \\
\quad+\int_{t}^{s} a(\tau, \omega) d \tau \int_{t}^{s} b l^{i}(\sigma, \omega) d \beta^{l}(\sigma, \omega) \\
\quad+\int_{t}^{s} b_{k}^{i}(\tau, \omega) d \beta(\tau, \omega) \int_{t}^{s} b_{b^{i}}(\sigma, \omega) d \beta^{l}(\sigma, \omega),
\end{aligned}
$$

from which follows (3.14) at once by virtue of Lemmas 2, 3 and 4 .

Lemma 6. Let $\hat{\varsigma}^{i}(t, \omega), i=1,2, \ldots, n$, be determined as in Theorem 6. For the point-system:

$$
\Delta: t=t_{0}<t_{1}<\ldots<t_{m}=s
$$

we put

$$
S(\Delta, \omega)=\sum_{\mu=1}^{m}\left|\hat{\xi}^{i}\left(t_{\mu}, \omega\right)-\xi^{i}\left(t_{\mu-i}, \omega\right)\right|\left|\xi^{j}\left(t_{\mu}, \omega\right)-\hat{\xi}^{j}\left(t_{\mu-1}, \omega\right)\right| .
$$

Then there exists $M=M(\varepsilon)$ independent of $\Delta$ for any $\varepsilon>0$ such that

$$
\operatorname{Pr}\left\{\omega \in \Omega_{1}, S(A, \omega)>M\right\}<\varepsilon \text {. }
$$

Proof. We may consider the case that

$$
\int_{\Omega} \int_{t}^{s} b_{k}^{p}(\tau, \omega) d t P(d \omega)<\infty, \quad p=i, j, \quad k=1,2, \ldots, n .
$$

since, if our Lemma is established in this case, we can easily deduce our Lemma in the general case by the definition of stochastic integral. When there is no confusion, we omit the time parameter and the probability parameter (') in the following.

$$
\begin{aligned}
\left(\xi^{i}\left(t_{\mu}\right)-\xi^{i}\left(t_{\mu-1}\right)\right)\left(\xi^{j}\left(t_{\mu}\right)-\xi^{j}\left(t_{\mu-1}\right)\right) & \\
\quad & \int_{t_{\mu-1}}^{t_{\mu}} a^{i} d \tau \int_{t_{\mu-1}}^{t_{\mu}} a^{j} d \sigma+\int_{t_{\mu-1}}^{t_{\mu}} b_{k}^{i} d \beta^{k} \int_{t_{i \mu-1}}^{t_{\mu}} a^{j} d \sigma \\
& +\int_{t_{\mu-1}}^{t_{\mu}} a^{i} d \tau \int_{t_{\mu-1}}^{t_{\mu}} b_{k}^{i} d \beta^{k}+\int_{t_{i \mu-1}}^{t_{\mu}} b_{k}^{i} d \beta^{k} \int_{t_{\mu-1}}^{t_{\mu}} b_{k}^{j} d \beta^{k}
\end{aligned}
$$

Since

$$
S_{1}(\Delta, \omega) \equiv \sum_{\mu}\left|\int_{t_{\mu-1}}^{t_{\mu}} a^{i} d \tau\right| \int_{t_{\mu-1}}^{t_{\mu}} a^{j} d \sigma\left|\leqq \max _{t \leqq t^{\prime} \leqq s^{\prime} \leqq s} \int_{t^{\prime}}^{s^{\prime}} a^{i} d \tau \int_{t}^{s}\right| a^{j} \mid d \sigma
$$

for almost all $\omega \in \Omega_{1}$ we may find $M_{1}(s)$ independent of $d$ for any $\lesssim<0$ such that $M>M_{1}(\varepsilon)$ implies

$$
\operatorname{Pr}\left\{\omega \in \Omega_{1}, S_{1}(d, \omega)>M\right\}<\varepsilon / 4 .
$$

By the same way we may find $M_{2 k}(\xi)$ and $M_{3 k}(\xi)$ independent of $d$ for any 
$\varepsilon<0$ such that $M>M_{2 k}(\varepsilon)$ or $M>M_{3 k}(\varepsilon)$ implies

$$
\operatorname{Pr}\left\{\omega \in \Omega_{1}, S_{2 k}(\Delta, \omega)>M\right\}<\varepsilon / 4 r \text { or } \operatorname{Pr}\left\{\omega \in \Omega_{2}, S_{3 k}(\Delta, \omega)>M\right\}<\varepsilon / 4 r
$$
respectively, where

$$
\begin{aligned}
& S_{2 k}(\Delta, \omega)=\sum_{\mu} \mid \int_{t_{\mu-1}}^{t_{\mu}} b_{k}^{i} d \beta^{k} \| \int_{t_{\mu-1}}^{t_{\mu}} a^{j} d \sigma \\
& S_{3 k}(\Delta, \omega)=\sum_{\mu}\left\{\int_{t_{\mu-1}}^{t_{\mu}} a^{i} d \tau|| \int_{t_{\mu-1}}^{t_{\mu}} b_{k}^{j} d \beta^{k}\right\} .
\end{aligned}
$$

Put

$$
\begin{aligned}
& S_{4 k l} l(\Delta, \omega) \equiv \sum_{\mu}\left|\int_{t_{\mu-1}}^{t_{\mu}} b_{k}^{i} d \beta^{k}\right| \int_{t_{\mu-1}}^{t_{\mu}} b_{l}^{j} d \beta^{l} \mid=\sum_{\mu} A_{\mu} B_{\mu} . \\
& \int S_{4 k l}(\Delta, \omega) P(d \omega)=\sum_{\mu} \int_{\Omega}\left|A_{\mu}\right|^{2} P(d \omega)+\sum_{\mu} \int_{\Omega}\left|B_{\mu}\right|^{2} P(d \omega) \\
& \leqq \sum_{\mu} \int_{\Omega} \int_{t_{\mu-1}}^{t_{\mu}}\left(b_{k}{ }^{i}\right)^{2} d \tau P(d \omega)+\sum_{\mu} \int_{\Omega} \int_{t_{\mu-1}}^{t_{\mu}}\left(b_{l}^{j}\right)^{2} d \tau P(d \omega) \\
& \leqq \int_{\Omega} \int_{t}^{s}\left(b_{k}^{i}\right)^{2} d \tau P(d \omega)+\int_{\Omega} \int_{t}^{s}\left(b_{l}^{j}\right)^{2} d \sigma P(d \omega) .
\end{aligned}
$$

Thus we may find $M_{4 k l}(\varepsilon)$ independent of $\Delta$ for any $\varepsilon>0$ such that $M_{>}>M_{4 k l}(\varepsilon)$ implies

$$
\operatorname{Pr}\left\{\omega \in \Omega_{1}, S_{4 k l}(\Delta, \omega)>M\right\}<\varepsilon / 4 r^{2} .
$$

Since

$$
S(\Delta, \omega) \leqq S_{1}(\Delta, \omega)+\sum_{k} S_{2 k}(\Delta, \omega)+\sum_{k} S_{3 k}(\Delta, \omega)+\sum_{k, i} S_{4 k l}(\Delta, \omega),
$$

we have (3.15) by putting

$$
M=M(\varepsilon)=M_{1}(\varepsilon)+\sum_{k} M_{2 k}(\varepsilon)+\sum_{k} M_{3 k}(\varepsilon)+\sum_{k, l} M_{4 k l}(\varepsilon) .
$$

Proof of Theorem 6. By Taylor expansion of $f\left(t, x^{1}, \ldots, x^{n}\right)$ we have (the probability parameter $\omega$ being omitted)

$$
\begin{aligned}
\eta(s, \omega) & -\eta(t, \omega) \\
& =\sum_{k=1}^{m}\left(\eta\left(t_{k}^{m}, \omega\right)-\eta\left(t_{k-1}^{m}, \omega\right)\right) \quad\left(t_{k}^{m}=t+\frac{k}{m}(s-t)\right) \\
& =\sum_{k=1}^{m}\left[f(* k)\left(t_{k}^{m}-t_{k-1}^{m}\right)+\sum_{i=1}^{n} f_{i}(* k)\left(\xi^{i}\left(t_{k}^{m}\right)-\xi^{i}\left(t_{k-1}^{m}\right)\right)\right. \\
& +\frac{1}{2} \sum_{i j} f_{i j}\left({ }^{*} k\right)\left(\xi^{i}\left(t_{k}^{m}\right)-\xi^{i}\left(t_{k-1}^{m}\right)\right)\left(\xi^{j}\left(t_{k}^{m}\right)-\xi^{j}\left(t_{k-1}^{m}\right)\right) \\
& \left.+\sum_{i j} \theta_{i j k}^{m}\left(\xi^{i}\left(t_{k}^{m}\right)-\xi^{i}\left(t_{k-1}^{m}\right)\right)\left(\xi^{j}\left(t_{k}^{m}\right)-\xi^{j}\left(t_{k-1}^{m}\right)\right)\right],
\end{aligned}
$$

where

$$
{ }^{*} k=\left(t_{k-1}^{m}, \xi^{1}\left(t_{k-1}^{m}\right), \ldots, \xi^{n}\left(t_{k-1}^{m}\right)\right) .
$$


Since $f_{i j}\left(t, x^{1}, \ldots, x^{n}\right)$ are continuous and $\xi^{i}(t, \omega), i=1,2, \ldots, n$ are all continuous in $t$ for almost all $\omega \in \Omega_{1}, \theta_{i, j, k}^{m}$ tends to 0 uniformly in $m$ and $k$ as $n \rightarrow \infty$ for almost all $\omega \in \Omega_{1}$. Therefore we have

$$
\sum_{k i j} \theta_{i j k}^{m}\left({ }^{\prime \prime}\right)\left({ }^{\prime \prime}\right) \rightarrow 0 \text { (in probability on } \Omega_{1} \text { ) }
$$

by virtue of Lemma 6 .

By Lemma 5 the remainder equals the following expression:

$$
\begin{aligned}
& \int_{t}^{s}\left(f_{0}(*)+f_{i}(*) a^{i}(\tau)+\frac{1}{2} f_{i j}(*) b_{k}{ }^{i}(\tau) b_{k}{ }^{j}(\tau)\right) d \tau+\int_{t}^{s} f_{i}(*) b_{j}{ }^{i}(\tau) d \beta^{j}(\tau) \\
& +\frac{1}{2} \int_{t}^{s} f_{i j}\left(^{*}\right)\left[\left(\xi^{i}(\tau)-\xi^{i}\left(\lambda_{m}(\tau)\right)\right) a^{j}(\tau)+\left(\xi^{j}(\tau)-\xi^{j}\left(\lambda_{m}(\tau)\right)\right) a^{i}(\tau)\right] d \tau \\
& +\frac{1}{2} \int_{t}^{s} f_{i j}(*)\left[\left(\xi^{i}(\tau)-\xi^{i}\left(\lambda_{m}(\tau)\right)\right) b_{k}{ }^{j}(\tau)+\left(\xi^{j}(\tau)-\xi^{j}\left(\lambda_{m}(\tau)\right)\right) b_{k}^{i}(\tau)\right] d \beta^{k}(\tau),
\end{aligned}
$$

where $\lambda_{m}(\tau)$ denotes the maximum $t_{k}^{m}$ which dose not exceed $\tau$ and $*$ denotes $\left(\lambda_{m}(\tau), \xi^{1}\left(\lambda_{m}(\tau)\right), \ldots, \xi^{n}\left(\lambda_{m}(\tau)\right)\right)$.

But $\xi^{i}\left(\lambda_{n}(\tau), \omega\right) \rightarrow \xi^{i}(\tau, \omega)$ uniformly in $\tau$ for almost all $\omega \in \Omega_{1}$ as $n \rightarrow \infty$. Therefore, by letting $n \rightarrow \infty$ in (3.18) we obtain

$$
\begin{gathered}
\eta(s)-\eta(t)=\int_{t}^{s}\left(f_{0}(\tau, \xi)+f_{i}(\tau, \xi) a^{i}(\tau)+\frac{1}{2} f_{i j}(\tau, \xi) b_{k}^{i}(\tau) b_{k}^{j}(\tau)\right) d \tau \\
+\int_{t}^{s} f_{i}(\tau, \xi) b_{j}{ }^{i}(\tau) d \beta^{j}(\tau),
\end{gathered}
$$

which proves our Theorem 5 .

\section{REFERENCES}

[1] K. Itô: On stochastic differential equations in a differentiable manifold, this Journal Vol. 1, 1950.

[2] K. Itò: Stochastic differential equations, Memoris of the American Mathematical Society, 4, 1951.

Mathematical Institute, Nagoya University 
